\title{
Colour flux-tubes in static Pentaquark and Tetraquark systems
}

\author{
Pedro Bicudo, ${ }^{1}$ Nuno Cardoso, ${ }^{1}$ Marco Cardoso ${ }^{1}$ \\ ${ }^{1}$ CFTP, Instituto Superior Técnico, Universidade Técnica de Lisboa
}

October 31, 2018

\begin{abstract}
The colour fields created by the static tetraquark and pentaquark systems are computed in quenched SU(3) lattice QCD, with gauge invariant lattice operators, in a $24^{3} \times 48$ lattice at $\beta=6.2$. We generate our quenched configurations with GPUs, and detail the respective benchmanrks in different $\mathrm{SU}(\mathrm{N})$ groups. While at smaller distances the coulomb potential is expected to dominate, at larger distances it is expected that fundamental flux tubes, similar to the flux-tube between a quark and an antiquark, emerge and confine the quarks. In order to minimize the potential the fundamental flux tubes should connect at 120 o angles. We compute the square of the colour fields utilizing plaquettes, and locate the static sources with generalized Wilson loops and with APE smearing. The tetraquark system is well described by a double-Y-shaped flux-tube, with two Steiner points, but when quark-antiquark pairs are close enough the two junctions collapse and we have an X-shaped flux-tube, with one Steiner point. The pentaquark system is well described by a three-Y-shaped flux-tube where the three flux the junctions are Steiner points.
\end{abstract}

\section{Motivation}

Multiquark exotic hadrons like the tetraquark and the pentaquark, different from the the ordinary mesons and baryons, have been studied and searched for many years. The tetraquark was initially proposed by Jaffe [1] as a bound state formed by two quarks and two antiquarks. Presently several observed resonances are tetraquark candidates. The most recent tetraquark candidates have been reported by the Belle Collaboration in May, the charged bottomonium $Z_{b}^{+}(10610)$ and $Z_{b}^{+}(10650)[2$. However a better understanding of tetraquarks is necessary to confirm or disprove the X, Y and possibly also light resonances candidates as tetraquark states.

On the theoretical side, the first efforts have been to search for bound states below the strong decay threshold [3, 4, 5, 6], as it is apparent that the absence of a potential barrier may produce a large decay width to any open channel. Recent investigations found that the presence of an angular momentum centrifugal barrier may increase the stability of the system [7, 8].

In the last years, the static tetraquark potential has been studied in Lattice QCD computations [9, 10, 11. The authors concluded that when the quark-quark are well separated from the antiquarkantiquark, the tetraquark potential is consistent with One Gluon Exchange Coulomb potentials plus a four-body confining potential, suggesting the formation of a double-Y flux tube, as in Fig. 1], composed of five linear fundamental flux tubes meeting in two Fermat points [6, 12, 13]. A Fermat, or Steiner, point is defined as a junction minimizing the total length of strings, where linear individual strings join at $120^{\circ}$ angles. When a quark approaches an antiquark, the minimum potential changes to a sum of 


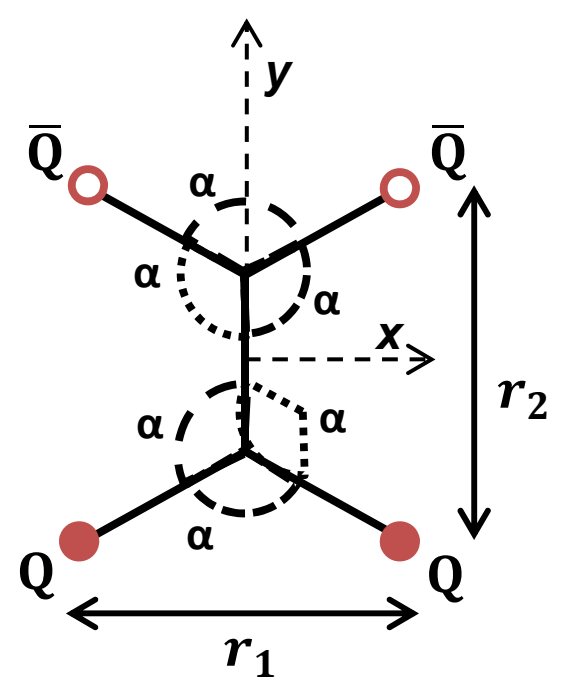

(a)

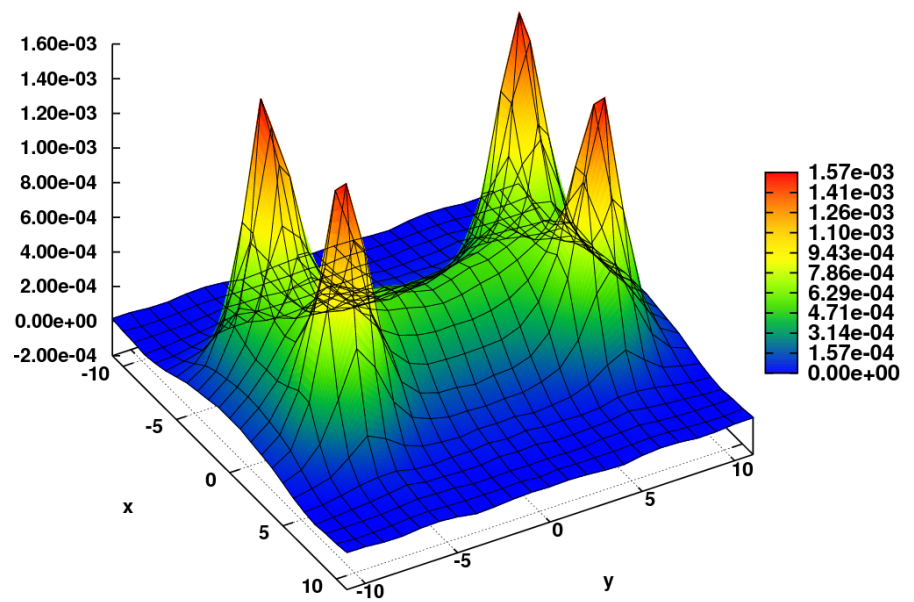

(b)

Figure 1: We compare (a) the tetraquark flux tube (or string) model, the elementary flux tubes meet in two Fermat points, at an angle of $\alpha=120^{\circ}$ to form a double-Y flux tube (except when this is impossible and the flux tube is X-shaped) with (b) Lagrangian density $3 \mathrm{D}$ plot for $r_{1}=8, r_{2}=14$, presented in lattice spacing units (colour online).

two quark-antiquark potentials, which indicates a two meson state. This is consistent with the triple flip-flop potential, minimizing the length, with either tetraquark flux tubes or meson-meson flux tubes, of thin flux tubes connecting the different quarks or antiquarks [6, 8].

Here we study the colour fields for the static tetraquark system [14], with the aim of observing the tetraquark flux tubes suggested by these static potential computations. The study of the colour fields in a tetraquark is important to discriminate between different multi-quark Hamiltonian models. Unlike the colour fields of simpler few-body systems, say mesons, baryons and hybrids, [15, 16, 17, 18], the tetraquark fields have not been previously studied in lattice QCD.

\section{Computing Fields with the Wilson loop and the Plaquette}

To impose a static tetraquark, we utilize the respective Wilson loop [9, 10] of Fig. 2, given by $W_{4 Q}=$ $\frac{1}{3} \operatorname{Tr}\left(M_{1} R_{12} M_{2} L_{12}\right)$, where

$$
\begin{aligned}
R_{12}^{a a^{\prime}} & =\frac{1}{2} \epsilon^{a b c} \epsilon^{a^{\prime} b^{\prime} c^{\prime}} R_{1}^{b b^{\prime}} R_{2}^{c c^{\prime}} \\
L_{12}^{a a^{\prime}} & =\frac{1}{2} \epsilon^{a b c} \epsilon^{a^{\prime} b^{\prime} c^{\prime}} L_{1}^{b b^{\prime}} L_{2}^{c c^{\prime}} .
\end{aligned}
$$

The chromoelectric and chromomagnetic fields on the lattice are given by the Wilson loop and plaquette expectation values,

$$
\begin{aligned}
\left\langle E_{i}^{2}(\mathbf{r})\right\rangle & =\left\langle P(\mathbf{r})_{0 i}\right\rangle-\frac{\left\langle W\left(r_{1}, r_{2}, T\right) P(\mathbf{r})_{0 i}\right\rangle}{\left\langle W\left(r_{1}, r_{2}, T\right)\right\rangle} \\
\left\langle B_{i}^{2}(\mathbf{r})\right\rangle & =\frac{\left\langle W\left(r_{1}, r_{2}, T\right) P(\mathbf{r})_{j k}\right\rangle}{\langle W\rangle\left(r_{1}, r_{2}, T\right)}-\left\langle P(\mathbf{r})_{j k}\right\rangle,
\end{aligned}
$$

where the $j k$ indices of the plaquette complement the index $i$ of the magnetic field, and where the plaquette at position $\mathbf{r}=(x, y, z)$ is computed at $t=T / 2$,

$$
P_{\mu \nu}(\mathbf{r})=1-\frac{1}{3} \operatorname{Re} \operatorname{Tr}\left[U_{\mu}(\mathbf{r}) U_{\nu}(\mathbf{r}+\mu) U_{\mu}^{\dagger}(\mathbf{r}+\nu) U_{\nu}^{\dagger}(\mathbf{r})\right] .
$$




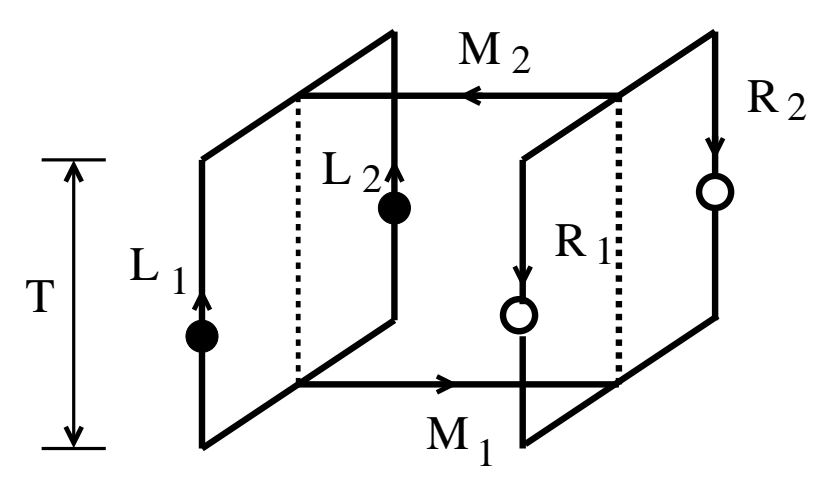

Figure 2: Tetraquark Wilson loop as defined by Alexandrou et al, and by Okiharu et al

The energy $(\mathcal{H})$ and lagrangian $(\mathcal{L})$ densities are then computed from the fields,

$$
\begin{aligned}
\langle\mathcal{H}(\mathbf{r})\rangle & =\frac{1}{2}\left(\left\langle\mathbf{E}^{2}(\mathbf{r})\right\rangle+\left\langle\mathbf{B}^{2}(\mathbf{r})\right\rangle\right), \\
\langle\mathcal{L}(\mathbf{r})\rangle & =\frac{1}{2}\left(\left\langle\mathbf{E}^{2}(\mathbf{r})\right\rangle-\left\langle\mathbf{B}^{2}(\mathbf{r})\right\rangle\right) .
\end{aligned}
$$

To produce the results presented in this work, we use 1121 quenched configurations in a $24^{3} \times 48$ lattice at $\beta=6.2$. We also test that these configurations are already close to the continuum limit in a larger, $32^{3} \times 64$ lattice. We present our results in lattice spacing units of $a$, with $a=0.07261(85) \mathrm{fm}$ or $a^{-1}=2718 \pm 32 \mathrm{MeV}$. We generate our configurations in NVIDIA GPUs of the FERMI series $(480,580$ and Tesla 2070) with a SU(3) CUDA code upgraded from our SU(2) combination of Cabibbo-Marinari pseudoheatbath and over-relaxation algorithm [19, 20].

To compute the static field expectation value, we plot the expectation value $\left\langle E_{i}^{2}(\mathbf{r})\right\rangle$ or $\left\langle B_{i}^{2}(\mathbf{r})\right\rangle$ as a function of the temporal extent $T$ of the Wilson loop. In order to improve the signal to noise ratio of the Wilson loop, we use 50 iterations of APE Smearing with $w=0.2$ (as in [17]) in the spatial directions and one iteration of hypercubic blocking (HYP) in the temporal direction. [21], with $\alpha_{1}=0.75, \alpha_{2}=0.6$ and $\alpha_{3}=0.3$. At sufficiently large $T$, the groundstate corresponding to the studied quantum numbers dominates, and the expectation value of the fields tends to a horizontal plateau. for each point $\mathbf{r}$ determined by the plaquette position. For the distances $r_{1}$ and $r_{2}$ considered, we find in the range of $T \in[3,12]$ in lattice units, horizontal plateaux with a $\chi^{2} /$ dof $\in[0.3,2.0]$. We finally compute the error bars of the fields with the jackknife method.

\section{The Tetraquark fields}

In our simulations, the quarks are fixed at $\left( \pm r_{1} / 2,-r 2 / 2,0\right)$ and the antiquarks at $\left( \pm r_{1} / 2, r_{2} / 2,0\right)$, with $r_{1}$ extending up to 8 lattice spacing units and $r_{2}$ extended up to 14 lattice spacing units, in order to include the relevant cases where $r_{2}>\sqrt{3} r_{1}$. Notice that in the string picture, at the line $r_{2}=\sqrt{3} r_{1}$ in our $\left(r_{1}, r_{2}\right)$ parameter space, the transition between the double-Y, or butterfly, tetraquark geometry in Fig. 1a to the meson-meson geometry should occur. The results are presented only for the $x y$ plane since the quarks are in this plane and the results with $z \neq 0$ are less interesting for this study. The flux tube fields can be seen in Fig. 1b, 3 and 4 . Theses figures exhibit clearly tetraquark double-Y, or butterfly, shaped flux tubes. The flux tubes have a finite width, and are not infinitely thin as in the string models inspiring the Fermat points and the triple flip-flop potential, but nevertheless the junctions are close to the Fermat points, thus justifying the use of string models for the quark confinement in constituent quark models. 


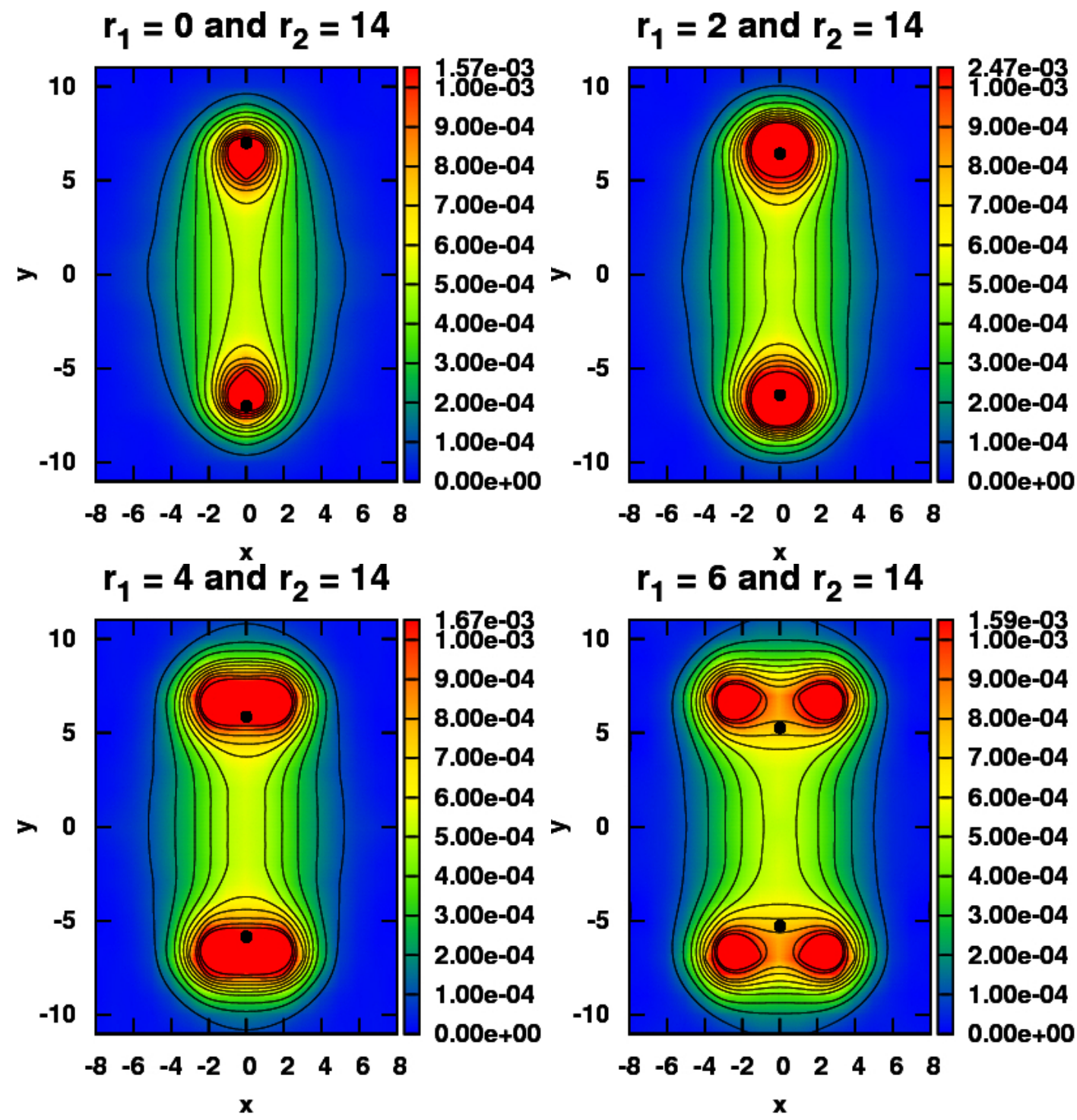

Figure 3: Lagrangian density for $r_{2}=14$ and $r_{1}$ from 0 to 6 . The black dot points correspond to the Fermat points. The results are presented in lattice spacing units (colour online).

In Fig. 5 (a), we plot the chromoelectric field along the central flux tube, $\left\langle E_{y}^{2}\right\rangle$ at $x=0$, for $r_{1}=8, r_{2}=14$. As expected, the chromoelectric field along $y$ is in agreement with the position of the Fermat points. The chromoelectric field along the $x=0$ central axis is maximal close to the Fermat points situated at $x \simeq-4.69$ and at $x \simeq 4.69$, flattens in the middle of the flux tube. Outside the flux tube, the chromoelectric field is almost residual.

In Fig. 5 (b), we compare the chromoelectric field for the tetraquark and the quark-antiquark system in the middle of the flux tube between the (di)quark and the (di)antiquark. As can be seen, for our larger distance $r_{2}=14$ where the source effects are small, the chromoelectric field is identical up to the error bars, and this confirms that the tetraquark flux tube is composed of a set of fundamental flux tubes with Fermat junctions.

To check which of the colour structures, tetraquark or meson-meson, produces the groundstate flux tube, we study the $\chi^{2} /$ dof of the $T$ plateaux. Surprisingly, event at distances as small as $r_{2} \simeq \frac{1}{2} r_{1} \sqrt{3}$, where the flip-flop potential favours the two-meson flux tube, we find $T$ plateaux with a good $\chi^{2} /$ dof. This shows that the mixing between the tetraquark flux tube and the meson-meson flux tube is small, and it is possible to study clear tetraquark flux tubes even at relatively small quark-antiquark distances. 


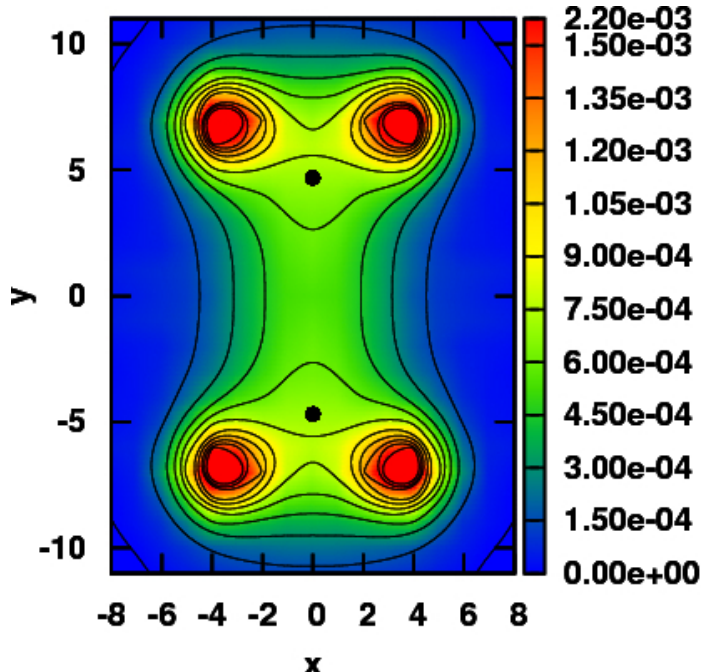

(a) $\left\langle E^{2}\right\rangle$

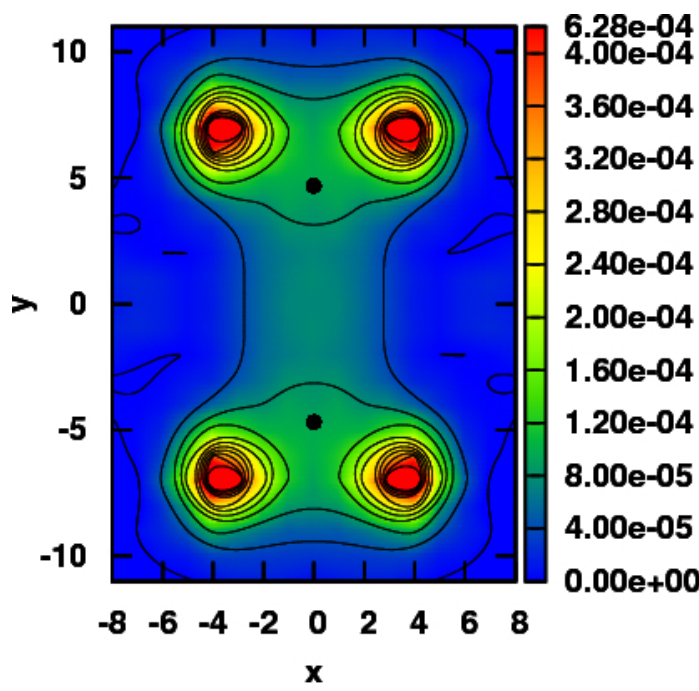

(c) Energy Density

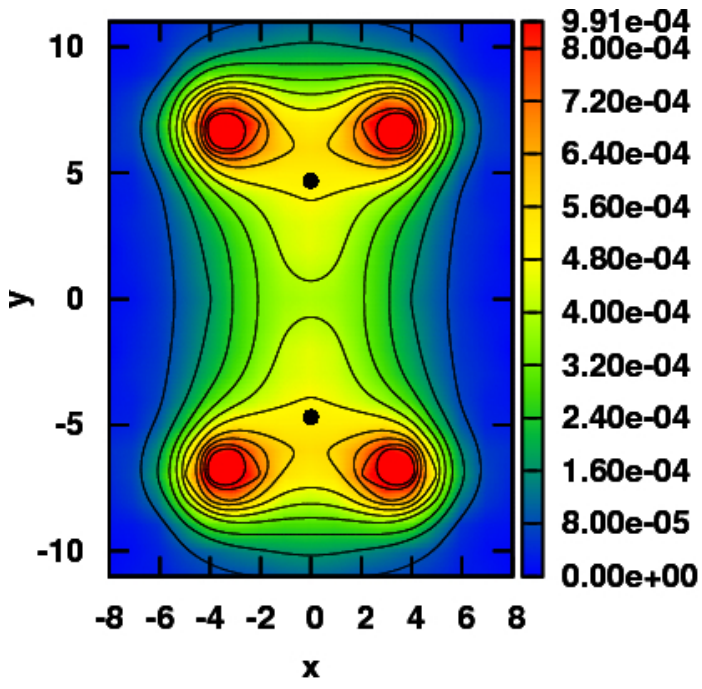

(b) $-\left\langle B^{2}\right\rangle$

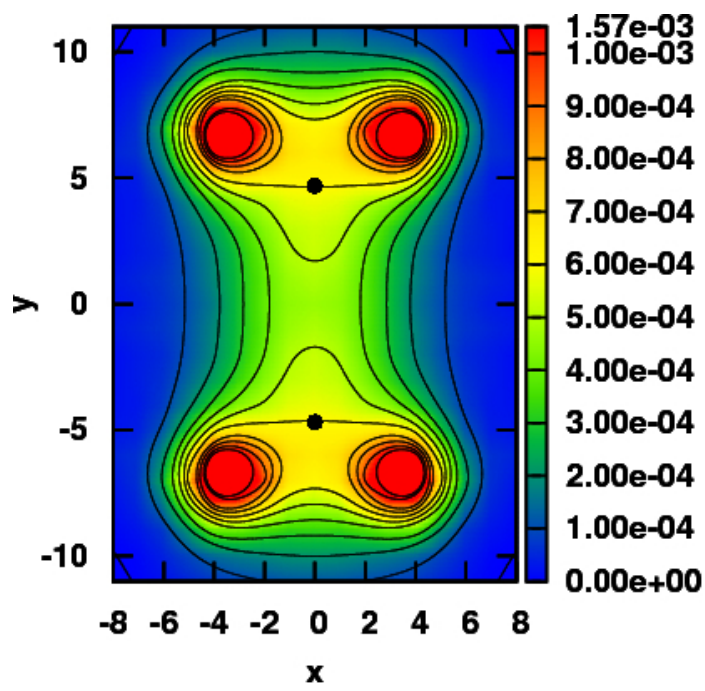

(d) Lagrangian Density

Figure 4: Colour fields, energy density and Lagrangian density for $r_{1}=8$ and $r_{2}=14$. The black dot points correspond to the Fermat points. The results are presented in lattice spacing units (colour online).

\section{Foreword}

- The flux tubes remain interesting in Lattice QCD, our results support the string model of confinement, in particular for the tetraquark static potential

- The mixing between the tetraquark and meson-meson flux-tubes is small, which may contribute for narrower tetraquark resonances.

- We are now studying in more detail other flux tubes, as for the pentaquark in Fig. 6.

- We will soon have codes for $\mathrm{SU}(2), \mathrm{SU}(3), \mathrm{SU}(4)$, etc, available on our webpage [19, 20].

This work was partly funded by the FCT contracts, PTDC/FIS/100968/2008, CERN/FP/109327/2009, CERN/FP/116333/2010 and CERN/FP/116383/2010. 

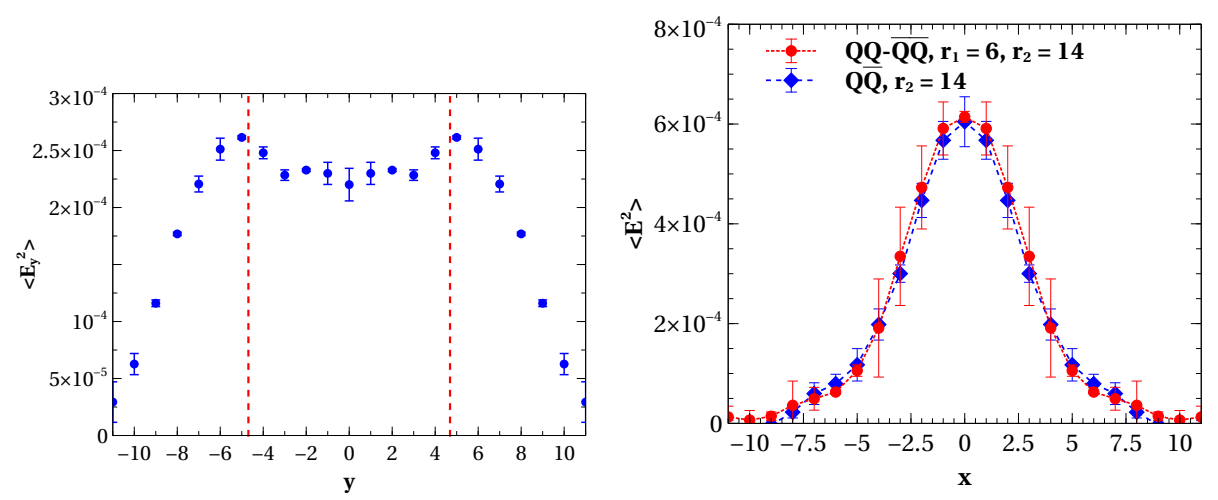

Figure 5: (a) $\left\langle E_{y}^{2}\right\rangle$ in the central axis $x=0$ for $r_{1}=8, r_{2}=14$. We show with vertical dashed lines the location of the two Fermat points. (b) Profile cut at $y=0$ of the chromoelectric field for the tetraquark and quark-antiquark systems in the middle of the flux tube. The results are presented in lattice spacing units (colour online).

Nuno Cardoso is also supported by FCT under the contract SFRH/BD/44416/2008.

\section{References}

[1] R. L. Jaffe, "Multi-Quark Hadrons. 1. The Phenomenology of (2 Quark 2 anti-Quark) Mesons," Phys. Rev., vol. D15, p. 267, 1977.

[2] B. Collaboration, "Observation of two charged bottomonium-like resonances," 2011.

[3] M. W. Beinker, B. C. Metsch, and H. R. Petry, "Bound $q^{* *} 2-$ anti- $q^{* *} 2$ states in a constituent quark model," J. Phys., vol. G22, pp. 1151-1160, 1996.

[4] S. Zouzou, B. Silvestre-Brac, C. Gignoux, and J. M. Richard, "FOUR QUARK BOUND STATES," Z. Phys., vol. C30, p. 457, 1986.

[5] B. A. Gelman and S. Nussinov, "Does a narrow tetraquark c c anti-u anti-d state exist?," Phys. Lett., vol. B551, pp. 296-304, 2003.

[6] J. Vijande, A. Valcarce, and J. M. Richard, "Stability of multiquarks in a simple string model," Phys. Rev., vol. D76, p. 114013, 2007.

[7] M. Karliner and H. J. Lipkin, "A Diquark-Triquark Model for the KN Pentaquark," Phys. Lett., vol. B575, pp. 249-255, 2003.

[8] P. Bicudo and M. Cardoso, "Tetraquark resonances with the triple flip-flop potential, decays in the cherry in a broken glass approximation," Phys. Rev., vol. D83, p. 094010, 2011.

[9] C. Alexandrou and G. Koutsou, "The static tetraquark and pentaquark potentials," Phys. Rev., vol. D71, p. 014504, 2005.

[10] F. Okiharu, H. Suganuma, and T. T. Takahashi, "The tetraquark potential and flip-flop in SU(3) lattice QCD," Phys. Rev., vol. D72, p. 014505, 2005.

[11] V. Bornyakov, P. Boyko, M. Chernodub, and M. Polikarpov, "Interactions of confining strings in $\mathrm{SU}(3)$ gluodynamics," 2005. 

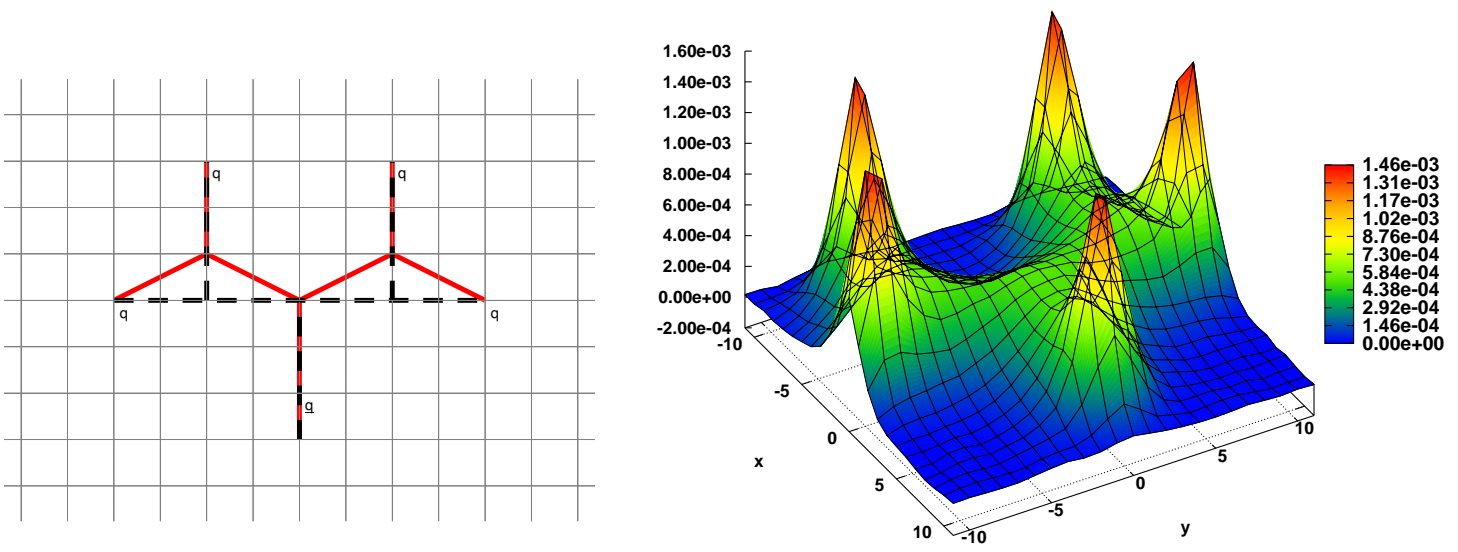

Figure 6: Wilson loop geometry, and preliminary result for the field, (Lagrangian density) for the static pentaquark. The results are presented in lattice spacing units (colour online).

[12] P. Bicudo and M. Cardoso, "Iterative method to compute the Fermat points and Fermat distances of multiquarks," Phys. Lett., vol. B674, pp. 98-102, 2009.

[13] J.-M. Richard, "Steiner-tree confinement and tetraquarks," 2009.

[14] N. Cardoso, M. Cardoso, and P. Bicudo, "Colour fields of the static hybrid gluon-quark-antiquark system," Acta Phys. Polon. Supp., vol. 3, pp. 997-1002, 2010.

[15] H. Ichie, V. Bornyakov, T. Streuer, and G. Schierholz, "Flux tubes of two- and three-quark system in full QCD," Nucl. Phys., vol. A721, pp. 899-902, 2003.

[16] F. Okiharu and R. M. Woloshyn, "An alternate smearing method for Wilson loops in lattice QCD," Eur. Phys. J., vol. C35, pp. 537-542, 2004.

[17] M. Cardoso, N. Cardoso, and P. Bicudo, "Lattice QCD computation of the colour fields for the static hybrid quark-gluon-antiquark system, and microscopic study of the Casimir scaling," Phys. Rev., vol. D81, p. 034504, 2010.

[18] N. Cardoso, M. Cardoso, and P. Bicudo, "Gauge invariant SU(3) lattice computation of the dual gluon mass and of the dual Ginzburg-Landau parameters $\lambda$ and $\xi$ in QCD," 2010.

[19] N. Cardoso and P. Bicudo, "SU(2) Lattice Gauge Theory Simulations on Fermi GPUs," J. Comput. Phys., vol. 230, pp. 3998-4010, 2011.

[20] PTQCD, 2011. the CUDA codes are available at Portuguese Lattice QCD collaboration, http: //nemea.ist.utl.pt/ ptqcd.

[21] A. Hasenfratz and F. Knechtli, "Flavor symmetry and the static potential with hypercubic blocking," Phys. Rev. D, vol. 64-3, p. 034504, 2001. 